\title{
On the possibility of track length based Monte-Carlo algorithms for stationary drift-diffusion systems with sources and sinks
}

\author{
D. Reiser, ${ }^{1}$ J. Romazanov, ${ }^{1}$ and Ch. Linsmeier $^{1}$ \\ ${ }^{1}$ Forschungszentrum Jülich GmbH, Institut für Energie- und Klimaforschung - Plasmaphysik, \\ Partner of the Trilateral Euregio Cluster (TEC), 52425 Jülich, Germany
}

\begin{abstract}
The problem of constructing Monte-Carlo solutions of drift-diffusion systems corresponding to Fokker-Planck equations with sources and sinks is revisited. Firstly, a compact formalism is introduced for the specific problem of stationary solutions. This leads to identification of the dwell time as the key quantity to characterize the system and to obtain a proper normalization for statistical analysis of numerical results. Secondly, the question of appropriate track length estimators for drift-diffusion systems is discussed for a $1 \mathrm{D}$ model system. It is found that a simple track length estimator can be given only for pure drift motion without diffusion. The stochastic nature of the diffusive part cannot be appropriately described by the path length of simulation particles. Further analysis of the usual situation with inhomogeneous drift and diffusion coefficients leads to an error estimate based on particle trajectories. The result for limits in grid cell size and time step used for the construction of Monte-Carlo trajectories resembles the Courant-FriedrichsLewy and von Neumann conditions for explicit methods.
\end{abstract}

Keywords: Monte-Carlo method; Fokker-Planck equation; drift-diffusion transport; track length estimator

\section{INTRODUCTION}

The Monte-Carlo approach is a well-known and widely used method for the numerical solution of timedependent and stationary problems [1-5]. Its particular strength is in the flexible incoporation of complex multi-dimensional geometries, its straightforward implementation in computer codes and its algorithmic robustness. Although sometimes guided by intuition, most applications are based on rigorous mathematical relations between systems of partial differential equations, corresponding Green's functions and stochastic processes. Many examples of such applications are based on FokkerPlanck models for drift-diffusion dynamics and its equivalent in the theory of stochastic differential equations. To mention only a few: codes in plasma physics research for studies on kinetic impurity transport [6-9], for neutral particle transport [10-12] and for plasma fluid transport [13] are based on certain variants of Fokker-Planck models including sources and sinks. Usually these codes are optimized for the treatment of stationary systems and all together they suffer from statistical noise due to limited CPU time which limits the number of stastistical samples. The search for a solution to this problem, i. e. increasing the algorithmic efficiency and reducing the noise, has a long history and led to the design of so-called estimators [14] which allow to gain more information out of the computational effort than simpler and perhaps more intuitive methods. One example of such a device is the track length estimator, which does not only accounts for certain locations of simulation particles, but rather considers the finite path length of simulation particles to evaluate the particle distributions in the computational volume and thereby reduces the statistical noise in the numerical solution. Although well established for many years in simulations of neutrons and neutral par- ticle transport it is surprising that its implementation in diffusion problems is not that well discussed nor documented. Questions in this context concern a proper normalization of numerical solutions, error estimates and a rigorous mathematical derivation of sometimes intuitively obvious methods. In this paper we want to contribute to the discussion of implementing a track length estimator in drift-diffusion problems closely related to the research fields mentioned above. To introduce notation and mathematical framework the problem at hand is sketched briefly in section II. The formalism leads immediately to the dwell time of the physical system as the key quantity for constructing a time stepping algorithm. In section III the general framework is applied to the Fokker-Planck equation. Well known facts on its corresponding stochastic differential equations and Green's functions are used to discuss the particular Monte-Carlo approach for its solution. Without loss of generality, basic results are derived for the particular case of a homogeneous 1D problem. Based on this, in section IV the concept of the track length as a mean to estimate the dwell time is discussed. The sobering result is that a straightforward use of a track length estimator is possible for the pure drift motion only. After a few general comments on simulations of diffusive motion, the analysis of the more important inhomogeneous 1D case in section $\mathrm{V}$ shows that requirements on accuracy of the stochastic approach leads essentially to restrictions of time step and spatial resolution recovering the CourantFriedrichs-Lewy and von Neumann conditions for explicit finite difference methods. In the concluding section VI a short summary of the findings is given. 


\section{CONSTRUCTION OF MONTE-CARLO PATHS, DWELL TIME AND NORMALIZATION}

In the context of particle transport, collision processes and fluid flow the Monte-Carlo approach can be considered roughly as a sampling of random paths of simulation particles according to certain rules determining the discrete steps in a computational volume. The distribution of these particles resulting from a given particle ensemble at the beginning of the simulation then represents the solution for a particular quantity of interest. A random path in configuration space can be described by a probability density $P_{m}^{+}\left(\mathbf{x}_{m} \mid \mathbf{x}_{0} ; t_{m}\right)$ representing a path of a single particle starting at location $\mathbf{x}_{0}$ and moving to location $\mathbf{x}_{m}$ via $m$ steps passing the intermediate locations $\mathbf{x}_{1}, \mathbf{x}_{2}, \ldots, \mathbf{x}_{m-1}$. The time corresponding to the step from $\mathbf{x}_{i-1}$ to $\mathbf{x}_{i}$ is denoted by $\Delta_{i}$, the total time needed for the entire path is denoted by $t_{m}=\sum_{i=1}^{m} \Delta_{i}$. Each step is a random event guided by some transition probability density $p^{+}\left(\mathbf{x}_{i} \mid \mathbf{x}_{i-1} ; \Delta_{i}\right)$. The notation $p^{+}$labels a step where the particle really arrives at $\mathbf{x}_{i}$, whereas the opposite event, where the particle disappears during the step, is taken into account by a transition probability $p^{-}\left(\mathbf{x}_{i} \mid \mathbf{x}_{i-1} ; \Delta_{i}\right)$. This takes into account processes like ionization, radioactive decay or absorption processes. The sum of those describes the certain event that the particle does a step. This is written as

$p\left(\mathbf{x}_{i} \mid \mathbf{x}_{i-1} ; \Delta_{i}\right)=p^{+}\left(\mathbf{x}_{i} \mid \mathbf{x}_{i-1} ; \Delta_{i}\right)+p^{-}\left(\mathbf{x}_{i} \mid \mathbf{x}_{i-1} ; \Delta_{i}\right)$

and

$\int_{\Omega} p\left(\mathbf{x}_{i} \mid \mathbf{x}_{i-1} ; \Delta_{i}\right) d \mathbf{x}_{i}=1$

and means that a particle existing at $\mathbf{x}_{i-1}$ will definitely do a step during a time increment $\Delta_{i}$ towards some location in the volume $\Omega$, but perhaps it will get lost. Therefore, the probability of a particle still existing after $m$ steps can be described by

$P_{m}^{+}\left(\mathbf{x}_{m} \mid \mathbf{x}_{0} ; t_{m}\right)=\prod_{i=1}^{m} p^{+}\left(\mathbf{x}_{i} \mid \mathbf{x}_{i-1} ; \Delta_{i}\right)$

The representation by a product of probabilities requires the Markov assumption, i. e. each step is independent of the previous steps. Therefore, the event that a particle does $m-1$ steps and gets lost at the $m$ th step is

$$
\begin{array}{r}
P_{m}^{-}\left(\mathbf{x}_{m} \mid \mathbf{x}_{0} ; t_{m}\right)=p^{-}\left(\mathbf{x}_{m} \mid \mathbf{x}_{m-1} ; \Delta_{m}\right) \times \\
\times P_{m-1}^{+}\left(\mathbf{x}_{m-1} \mid \mathbf{x}_{0} ; t_{m}-\Delta_{m}\right)
\end{array}
$$

Next the path probabilities are connected to ensembles of particles by assuming that the probability of finding a particle at $\mathbf{x}_{0}$ at time $t_{0}$ can be described by a probability distribution function $f^{+}\left(\mathbf{x}_{0}, t_{0}\right)$ obeying

$$
\int_{\Omega} f^{+}\left(\mathbf{x}_{0}, t_{0}\right) d \mathbf{x}_{0}=1
$$

The particle density $n$ of the ensemble at time $t_{0}$ is obtained by multiplication with the total number $N$ of particles in the volume $\Omega$

$n\left(\mathbf{x}_{0}, t_{0}\right)=N f^{+}\left(\mathbf{x}_{0}, t_{0}\right)$

Thus, the distribution function

$$
\begin{aligned}
f^{+} & \left(\mathbf{x}_{m}, t_{m}\right) \\
& =\int_{\Omega} \ldots \int_{\Omega} P_{m}^{+}\left(\mathbf{x}_{m} \mid \mathbf{x}_{0} ; t_{m}\right) f^{+}\left(\mathbf{x}_{0}, t_{0}\right) d \mathbf{x}_{m-1} \ldots d \mathbf{x}_{0}
\end{aligned}
$$

gives the distribution of particles $n\left(\mathbf{x}_{m}, t_{m}\right)=$ $N f^{+}\left(\mathbf{x}_{m}, t_{m}\right)$ in the volume after $m$ time steps. The resulting integral equation

$$
\begin{aligned}
f^{+} & \left(\mathbf{x}_{m}, t_{m}\right) \\
& =\int_{\Omega} p^{+}\left(\mathbf{x}_{m} \mid \mathbf{x}_{m-1} ; \Delta_{m}\right) f^{+}\left(\mathbf{x}_{m-1} ; t_{m-1}\right) d \mathbf{x}_{m-1}
\end{aligned}
$$

identifies the transition probabilities as propagators for the distribution function during the random path. To simplify notation and for subsequent discussions a general propagator for the Monte-Carlo chain is introduced by

$$
\begin{aligned}
G^{+} & \left(\mathbf{x}_{m} \mid \mathbf{x}_{j} ; t_{m}\right) \\
& =\int_{\Omega} \ldots \int_{\Omega} P_{m}^{+}\left(\mathbf{x}_{m} \mid \mathbf{x}_{j} ; t_{m}-t_{j}\right) d \mathbf{x}_{m-1} \ldots d \mathbf{x}_{j+1}
\end{aligned}
$$

This definition allows to write

$f^{+}\left(\mathbf{x}_{m}, t_{m}\right)=\int_{\Omega} G^{+}\left(\mathbf{x}_{m} \mid \mathbf{x}_{j} ; t_{m}-t_{j}\right) f^{+}\left(\mathbf{x}_{j}, t_{j}\right) d \mathbf{x}_{j}$

Similarly a propagator for the termination of the path is defined by

$$
\begin{aligned}
G^{-} & \left(\mathbf{x}_{m} \mid \mathbf{x}_{j} ; t_{m}\right) \\
& =\int_{\Omega} \ldots \int_{\Omega} P_{m}^{-}\left(\mathbf{x}_{m} \mid \mathbf{x}_{j} ; t_{m}-t_{j}\right) d \mathbf{x}_{m-1} \ldots d \mathbf{x}_{j+1}
\end{aligned}
$$

and a corresponding distribution function $f^{-}$

$f^{-}\left(\mathbf{x}_{m}, t_{m}\right)=\int_{\Omega} G^{-}\left(\mathbf{x}_{m} \mid \mathbf{x}_{j} ; t_{m}-t_{j}\right) f^{+}\left(\mathbf{x}_{j}, t_{j}\right) d \mathbf{x}_{j}$

which represents the loss probability distribution for particles at the $m$ th step. Due to the normalization condition of Eq. 2 a recursive relation for the distribution functions $f^{+}$and $f^{-}$at different times can be found

$$
\int_{\Omega} f^{+}\left(\mathbf{x}, t_{m}\right) d \mathbf{x}=\int_{\Omega} f^{+}\left(\mathbf{x}, t_{m-1}\right) d \mathbf{x}-\int_{\Omega} f^{-}\left(\mathbf{x}, t_{m}\right) d \mathbf{x}
$$


and it follows

$\int_{\Omega} f^{+}\left(\mathbf{x}, t_{m}\right) d \mathbf{x}+\sum_{i=1}^{m} \int_{\Omega} f^{-}\left(\mathbf{x}, t_{i}\right) d \mathbf{x}=1$

which simply expresses the fact that a particle is still present in the volume $\Omega$ after $m$ steps or was lost at an intermediate step. The distribution function $f^{-}(\mathbf{x}, t)$ can be used to derive certain statistical averages along the chain. For example, the average dwell time $\tau_{m}$ of particles along chains with $m$ steps, is given by

$\tau_{m}=\frac{\sum_{i=1}^{m} \int_{\Omega} t_{i} f^{-}\left(\mathbf{x}, t_{i}\right) d \mathbf{x}}{\sum_{i=1}^{m} \int_{\Omega} f^{-}\left(\mathbf{x}, t_{i}\right) d \mathbf{x}}=\frac{\sum_{i=1}^{m} \sum_{k=1}^{i} \Delta_{k} \int_{\Omega} f^{-}\left(\mathbf{x}, t_{i}\right) d \mathbf{x}}{\sum_{i=1}^{m} \int_{\Omega} f^{-}\left(\mathbf{x}, t_{i}\right) d \mathbf{x}}$

In appendix A the average dwell time $\tau$ for a system with finite Monte-Carlo chains is derived in the continuous limit. It is shown that the dwell time is given by the cumulative distribution function $F^{+}(\mathbf{x})$

$\tau=\int_{\Omega} \int_{0}^{\infty} f^{+}(\mathbf{x}, t) d t d \mathbf{x} \equiv \int_{\Omega} F^{+}(\mathbf{x}) d \mathbf{x}$

For completeness and to point out the relation to numerical procedures we show also the evaluation of Eq. 15 using discrete sums. However, in contrast to the continuous case, for this purpose it is necessary to require that all time steps are equal, i. e. $\Delta_{k}=\Delta$ and $t_{k}=k \Delta$ for $1 \leq k \leq m$. Then one can use Eq. 14 and the relations

$$
\begin{aligned}
& \sum_{i=1}^{m} \sum_{k=1}^{i} \int_{\Omega} f^{-}\left(\mathbf{x}, t_{k}\right) d \mathbf{x} \\
& =\sum_{i=1}^{m}(m+1-i) \int_{\Omega} f^{-}\left(\mathbf{x}, t_{i}\right) d \mathbf{x} \\
& =m-\sum_{i=1}^{m} \int_{\Omega} f^{+}\left(\mathbf{x}, t_{i}\right) d \mathbf{x}
\end{aligned}
$$

to find

$$
\begin{aligned}
& \frac{\tau_{m}}{\Delta} \sum_{i=1}^{m} \int_{\Omega} f^{-}\left(\mathbf{x}, t_{i}\right) d \mathbf{x} \\
&=(m+1) \sum_{i=1}^{m} \int_{\Omega} f^{-}\left(\mathbf{x}, t_{i}\right) d \mathbf{x} \\
&-m+\int_{\Omega} \sum_{i=1}^{m} f^{+}\left(\mathbf{x}, t_{i}\right) d \mathbf{x}
\end{aligned}
$$

Assuming now an upper limit $M$ for the number of Monte-Carlo steps, i. e. $f^{+}\left(\mathbf{x}, t_{m}\right)=0$ for $m \geq M$, one finds

$\tau_{M}=\Delta \int_{\Omega} \sum_{i=0}^{M} f^{+}\left(\mathbf{x}, t_{i}\right) d \mathbf{x} \equiv \Delta \int_{\Omega} F_{M}^{+}\left(\mathbf{x}, t_{M}\right) d \mathbf{x}$

Note, that the cumulative distribution function $F_{M}^{+}\left(\mathbf{x}, t_{M}\right)$ for the discrete case does not have the dimensions as its equivalent $F^{+}(\mathbf{x})$ in the continuous case, which is an integral over time. Actually, the Eq. 19 is the basic result to provide a link between the physical system and numerical models. To see this, the cumulative particle number

$N_{M}=\sum_{i=1}^{M} \int_{\Omega} n\left(\mathbf{x}, t_{i}\right) d \mathbf{x}=N \int_{\Omega} F_{M}^{+}\left(\mathbf{x}, t_{M}\right) d \mathbf{x}$

is considered, which represents the number of particles in the stationary system, where all path lengths are present simultaneously. On the other hand, this reflects the outcome of standard numerical approaches where a number of $N^{*}\left(t_{0}\right)$ particles is launched at time $t_{0}$ and their particular trajectories are tracked via markers at their respective positions at each time step $\Delta$ until they are lost, i. e. reached a maximum number of steps. The term markers just denotes the actual position of a particle and its tracking is usually done by increasing a counter by one for each grid cell in the discretized computational domain. Consequently, this means that the sum of all the markers for all particles $N_{M}^{*}=\sum_{i=1}^{M} N^{*}\left(t_{i}\right)$ in the simulation (labeled by the asterisk) is an estimate for the particle number $N_{M}$ in the stationary situation. Now it is to be noted that the dwell time $\tau_{M}$ is an intrinsic characteristic of the physical system under consideration and it is linked to the ratio of stationary particle number $N_{M}$ and source feeding via $N$ particles per time $\Delta$. This leads to another form of the basic result of Eq. 19:

$\frac{\tau_{M}}{\Delta}=\frac{N_{M}}{N}=\frac{N_{M}^{*}}{N^{*}}$

Indeed, the Eq. 21 provides the necessary normalization condition for the analysis of Monte-Carlo chains of stationary systems: the number of physical particles $N_{M}$ can be obtained by the simple normalization $N_{M} / N=N_{M}^{*} / N^{*}$, where $N$ is given by the known physical source strength, $N^{*}$ is the number of simulation particles and $N_{M}^{*}$ is the outcome of the numerical construction and superposition of Monte-Carlo paths. This approach is straightforward and gives accurate results as long as the chosen particle number and step size is sufficient to obtain good statistical estimates. This is quite often not easy to ensure. For this reason in the next section the marker method, where the dwell time is computed directly, will be compared with the track length method, which has been proven to be more efficient in some applications $[10 ; 14]$. But, here we will focus on its possible application for drift-diffusion systems described by Fokker-Planck models. 


\section{FOKKER-PLANCK EQUATION AND GREEN'S FUNCTIONS}

The multi-dimensional Fokker-Planck equation with sources and sinks can be written as

$\frac{\partial f}{\partial t}=-\nabla \cdot\left[\mathbf{V} f-\frac{1}{2} \nabla \cdot(\mathbf{D} f)\right]+S_{+}-S_{-}$

It describes the temporal evolution of a scalar function $f$ transported by a convection with drift velocity $\mathbf{V}$ and conduction guided by a diffusion tensor D. Sources are summarized by $S_{+}$and sinks by $S_{-}$, respectively. The Fokker-Planck equation naturally appears in the context of passive scalar transport like Brownian motion and, even though it is a linear equation, it plays an important role in linearized methods for non-linear fluid dynamics and similar problems. Here we consider Eq. 22 as an evolution equation for the particle distribution function $f^{+}$discussed in the previous section, but this does not affect any other interpretation. A very appealing feature of the Fokker-Planck equation is that for constant $\mathbf{V}$ and D its Green's function is known and that the importance of Fokker-Planck models for stochastic processes has been studied intensively. We refer to these fundamentals just by recalling two basic facts: (1) The Green's function for Eq. 22 without sources and sinks and constant $\mathbf{V}$ and $\mathbf{D}$ is given by

$G(\mathbf{x}, t)=\sqrt{\frac{\left|\mathbf{D}^{-1}\right|}{8 \pi^{3} t}} \exp \left[-\frac{(\mathbf{x}-\mathbf{V} t) \cdot \mathbf{D}^{-1} \cdot(\mathbf{x}-\mathbf{V} t)}{2 t}\right]$

and $G(\mathbf{x}, t)$ fulfills the integral relation

$f(\mathbf{x}+\Delta \mathbf{x}, t+\Delta t)=\int_{\Omega} G(\Delta \mathbf{x}, \Delta t) f(\mathbf{x}, t) d \mathbf{x}$

(2) The basic recipe for constructing a Monte-Carlo chain to obtain a discrete representation of a solution of Eq. 22 reads

$\Delta \mathbf{x}=\mathbf{V} \Delta t+\mathbf{B} \cdot \boldsymbol{\xi} \sqrt{\Delta t}$

where $\boldsymbol{\xi}$ is a vector whose components are three independent Gaussian random numbers with mean 0 and variance 1. The tensor $\mathbf{B}$ fulfills $\mathbf{D}=\mathbf{B} \cdot \mathbf{B}^{\mathrm{T}}$. The basic correspondence between these two aspects is that the Green's function can be regarded as the probability distribution of the step $\Delta \mathbf{x}$ and the construction rule of Eq. 25 provides exactly this Gaussian distribution. For details on this a vast amount of literature exists, see e. g. [15; 16] and references therein.

As for us, we continue with the discussion of the dwell time $\tau_{m}$ introduced in the previous section as the basic quantity for the evaluation of Monte-Carlo chains. For this purpose and to keep the math as simple as possible we restrict ourselves on a prototypical $1 \mathrm{D}$ problem

$\frac{\partial f}{\partial t}=-\frac{\partial}{\partial x}(V f)+\frac{1}{2} \frac{\partial^{2}}{\partial x^{2}}(D f)-\alpha f+S_{+}-S_{-}$
Here, $V$ and $D$ are constant drift and diffusion coefficients, $S_{+}$and $S_{-}$are unspecified source and sink terms and $\alpha$ is a decay rate introducing an additional loss process, e. g. ionization processes, radioactive decay or absorption processes. It is a strength of the Monte-Carlo method, that usually the source and sink terms $S_{+}$and $S_{-}$can be treated by starting and terminating MonteCarlo chains at particular locations. Therefore, we exclude the sources and sinks contained in $S_{+}$and $S_{-}$in the temporal evolution of $f$. The remaining transport piece and decay process can be computed via

$f(x, t+\Delta)=\int_{-\infty}^{\infty} G(x-y, \Delta) f(y, t) d y$

where

$G(x-y, \Delta)=\frac{e^{-\alpha \Delta}}{\sqrt{2 \pi D \Delta}} \exp \left[-\frac{(x-y-V \Delta)^{2}}{2 D \Delta}\right]$

is the relevant Green's function of Eq. 26 with properties

$\int_{-\infty}^{+\infty} G(x-y, \Delta) d x=\int_{-\infty}^{+\infty} G(x-y, \Delta) d y=e^{-\alpha \Delta}$

and

$\int_{-\infty}^{\infty} G\left(x-y, \Delta_{2}\right) G\left(y-z, \Delta_{1}\right) d y=G\left(x-z, \Delta_{1}+\Delta_{2}\right)$

Comparison of Eq. 27 with Eq. 8 shows that the Green's function $G(x-y, \Delta)$ can be regarded as the transition probability density $p^{+}(x \mid y ; \Delta)$ guiding the MonteCarlo steps. Similarly the loss probability is given by $p^{-}(x \mid y ; \Delta)=\left(1-e^{-\alpha \Delta}\right) G(x-y, \Delta)$. Note, that for pure drift motion the Green's function becomes

$p^{+}(x \mid y ; \Delta)=G(x-y, \Delta)=\delta(x-y-V \Delta) e^{-\alpha \Delta}$

with $\delta(x-y-V \Delta)$ being the Dirac delta function. Now we are in the position to evaluate certain integrals of section II. One finds

$\int_{-\infty}^{\infty} f^{+}\left(x, t_{m}\right) d x=e^{-\alpha t_{m}}$

and

$\int_{-\infty}^{\infty} f^{-}\left(x, t_{m}\right) d x=\left(e^{-\alpha \Delta_{m}}-1\right) e^{-\alpha t_{m}}$

The dwell time $\tau_{m}$ for the $1 \mathrm{D}$ problem reads

$\tau_{m}=\frac{\sum_{i=1}^{m} \sum_{k=1}^{i} \Delta_{k} \int_{-\infty}^{\infty} f^{-}\left(x, t_{i}\right) d x}{\sum_{i=1}^{m} \int_{-\infty}^{\infty} f^{-}\left(x, t_{i}\right) d x}$ 
and in the continuous limit with measure $\mu(t)=1-e^{\alpha t}$ (see appendix A) this provides the compact result

$$
\tau_{m}=\frac{\int_{0}^{t_{m}} \alpha t e^{-\alpha t} d t}{\int_{0}^{t_{m}} \alpha e^{-\alpha t} d t}=\frac{1}{\alpha}-\frac{t_{m} e^{-\alpha t_{m}}}{1-e^{-\alpha t_{m}}}
$$

Now we come to the question of average track length $\lambda_{m}$. As mentioned above, the reason to use this quantity in estimating a particle distribution is - as for any other choice of estimator [14] - to get more information from the time stepping and to use a larger time step. We start with the general discrete expression similar to the definition of $\tau_{m}$ in Eq. 15

$$
\lambda_{m}=\frac{\sum_{i=1}^{m} \int_{\Omega} \sum_{k=1}^{i}\left|\mathbf{x}_{k}-\mathbf{x}_{k-1}\right| f^{-}\left(\mathbf{x}_{k}, t_{i}\right) d \mathbf{x}_{k}}{\sum_{i=1}^{m} \int_{\Omega} f^{-}\left(\mathbf{x}, t_{i}\right) d \mathbf{x}}
$$

For the prototypical 1D problem considered here one finds by the use of Eqs. 27-30 that

$$
\begin{aligned}
& \int_{-\infty}^{\infty}\left|x_{k}-x_{k-1}\right| f^{-}\left(x_{k}, t_{i}\right) d x_{k} \\
& \quad=L\left(\Delta_{k}\right) \int_{-\infty}^{\infty} f^{-}\left(x, t_{i}\right) d x \quad \text { for } 1 \leq k \leq i
\end{aligned}
$$

where $L(\Delta)$ is the average length of a jump for a driftdiffusive time step $\Delta$

$$
\begin{aligned}
L(\Delta) & =\int_{-\infty}^{\infty} \frac{|x|}{\sqrt{2 \pi D \Delta}} \exp \left[-\frac{(x-V \Delta)^{2}}{2 D \Delta}\right] d x \\
& =\sqrt{\frac{2 D \Delta}{\pi}} \exp \left(-\frac{V^{2} \Delta}{2 D}\right)+V \Delta \operatorname{erf}\left(V \sqrt{\frac{\Delta}{2 D}}\right)
\end{aligned}
$$

Thus, the average track length $\lambda_{m}$ in the $1 \mathrm{D}$ case is given as

$$
\lambda_{m}=\frac{\sum_{i=1}^{m} \sum_{k=1}^{i} L\left(\Delta_{k}\right) \int_{-\infty}^{\infty} f^{-}\left(x, t_{i}\right) d x}{\sum_{i=1}^{m} \int_{-\infty}^{\infty} f^{-}\left(x, t_{i}\right) d x}
$$

Note that $L\left(\Delta_{k}\right) \rightarrow|V| \Delta_{k}$ for $D \rightarrow 0$ and then $\lambda_{m}=$ $|V| \tau_{m}$, and this limit forms the basis for the use of track length in many applications with pure drift motion: without any diffusion the average track length is synonymous with the dwell time and this fact gives reason to sample path increments instead of counting markers during the construction of Monte-Carlo path. Of course an increase of the numerical efficiency has to be checked for the specific problem at hand. Sometimes the simple marker method might be more efficient, even if smaller time steps are necessary. However, for finite diffusion coefficient $D>0$ the extension of the track length method encounters certain problems which will be discussed in more detail in the next section.

\section{DWELL TIME VS TRACK LENGTH}

In section II the dwell time has been shown to be the basic quantity for normalizing and evaluating numerical Monte-Carlo chains for stationary distributions. This led to the normalization condition Eq. 21. For a 1D driftdiffusion system expressions have been derived for the dwell time $\tau_{m}$ (Eq. 34) and the average path length $\lambda_{m}$ (Eq. 39). The question initiating the present analysis was: by which means the track length $\lambda_{m}$ might be useful in replacing the time consuming computation of the dwell time $\tau_{m}$ by markers. The marker method gives the right results, but it relies on the assumption that the simulation jump is entirely contained in the grid cell where the counter is increased by one. If cell faces are crossed this is not the case and some error might occur in the weighting of the particles. On the other hand the efficiency of the algorithm might be increased by analyzing the details of the path between two successive locations, e. g. by a proper splitting of the track length and distributing appropriate weights. At first glance and based on the results of section III the answer might look simple: due to the fact that $L\left(\Delta_{i}\right)$ is known analytically and that it depends only on the time step $\Delta_{i}$ (the case, where $L\left(\Delta_{i}\right)$ also varies spatially, will be part of the discussion in the next section) the comparison of Eqs. 15 and 34 with Eqs. 36 and 39 leads to the idea that it might be enough to sample the scaled path

$\Phi_{i}=\frac{\left|x_{i}-x_{i-1}\right|}{L\left(\Delta_{i}\right)} \Delta_{i}$

because Eqs. 34 and 37 give for the chain average of $\Phi_{i}$ immediately

$$
\frac{\sum_{i=1}^{m} \int_{-\infty}^{\infty} \sum_{k=1}^{i} \frac{\left|x_{k}-x_{k-1}\right|}{L\left(\Delta_{k}\right)} \Delta_{k} f^{-}\left(x, t_{i}\right) d x}{\sum_{i=1}^{m} \int_{-\infty}^{\infty} f^{-}\left(x, t_{i}\right) d x}=\tau_{m}
$$

As noted before, for pure drift motion the average step length is just $L\left(\Delta_{k}\right)=|V| \Delta_{k}$. Therefore, the numerical procedure of sampling $\Phi_{i}$ consists of sampling $\left|x_{i}-x_{i-1}\right| /|V|$ for all particles and all paths, i. e. the time needed to move along straight line segments with 
velocity $|V|$. Then it is clear that the resulting cumulative distribution represents the dwell time. For a diffusive step it is not that obvious due to the random character of the step expressed by the construction of Eq. 25, which reads in the $1 \mathrm{D}$ case

$\Delta x=V \Delta t+\sqrt{D \Delta t} \gamma$

Here, $\gamma$ is a Gaussian random number with zero mean and variance 1 . Indeed, the special nature of the FokkerPlanck model does not allow to introduce an effective velocity $L(\Delta) / \Delta$ to be used to scale the step length $\left|x_{i}-x_{i-1}\right|$. The problem is illustrated by Fig. 1 for a particle passing two cell faces. The advantage of a track length estimator would lie in the possibility to evaluate pieces of a single particle step when particles jump over more than one grid cell in the computational domain. The grid cells are just serving for counting markers during Monte-Carlo time stepping and to obtain a discrete representation of the solution of interest. Actually, the idea is to distribute dwell times to different grid cells according to a proper splitting of the distance between to subsequent locations, i.e. $\Delta=\Delta_{1}+\Delta_{2}$. This would allow large time steps, because the single step is retroactively splitted into several steps. Therefore, having computed

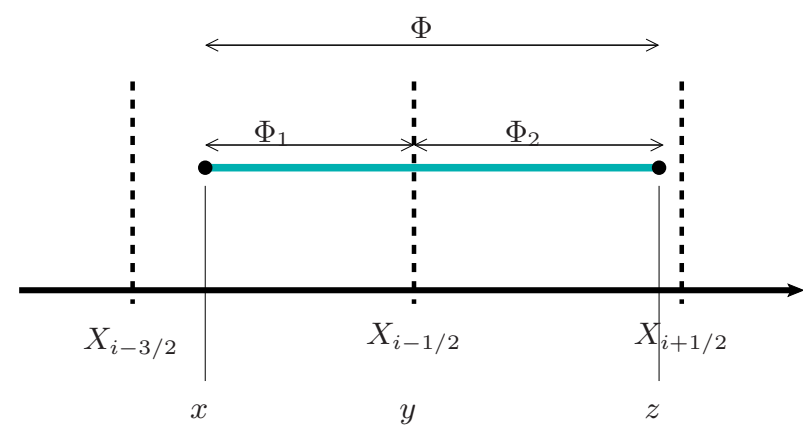

FIG. 1 Sketch of a particle step during time step $\Delta$ from location $x$ to location $z$. The cell boundary at $y=X_{i-1 / 2}$ is passed within a single step. An appropriate estimator $\Phi$ would cover the entire step. The evaluation of the MonteCarlo trajectories needs the determination of the components $\Phi_{1}$ and $\Phi_{2}$ for proper sampling.

a step $x \rightarrow z$ for a given time step $\Delta$ which passes e. g. a single grid cell boundary at point $y$, a unique splitting of the estimator $\Phi$ would be needed to evaluate

$\Phi=\frac{|z-x|}{L(\Delta)} \Delta=\frac{|y-x|}{L\left(\Delta_{1}\right)} \Delta_{1}+\frac{|z-y|}{L\left(\Delta_{2}\right)} \Delta_{2}=\Phi_{1}+\Phi_{2}$

However, considering just the symmetric case $|z-y|=\mid y-$ $x \mid$, where one requires $\Delta_{1}=\Delta_{2}$ and $L(\Delta)=L\left(\Delta_{1}\right)+L\left(\Delta_{2}\right)$, illustrates that Eq. 43 can not be fulfilled in the general drift-diffusive case. For the pure drift motion with $D=0$ this is always possible, but unfortunately one finds by inspection of Eq. 38 that for finite diffusion coefficient $D$

$L\left(\Delta_{1}\right)+L\left(\Delta_{2}\right)>L\left(\Delta_{1}+\Delta_{2}\right)$ for any finite $\Delta_{1}$ and $\Delta_{2}$. Moreover, when considering the sum of average path lengths for $m$ steps each corresponding to a time increment $\Delta$ one finds that

$$
\begin{gathered}
\sum_{k=1}^{m} L(k \Delta)=\sum_{k=1}^{m} \sqrt{\frac{2 D k \Delta}{\pi}} \exp \left(-\frac{V^{2} k \Delta}{2 D}\right) \\
+\sum_{k=1}^{m} V k \Delta \operatorname{erf}\left(V \sqrt{\frac{k \Delta}{2 D}}\right)
\end{gathered}
$$

is a monotonically increasing function of the number of steps $m$ if the total time $m \Delta$ is kept constant. The total length diverges for $m \rightarrow \infty$. Then $\sum_{k=1}^{m} L(k \Delta) \rightarrow \infty$ and this shows that the length $L(\Delta)$ is not a useful quantity to describe the path of a diffusing particle during a prescribed time interval $\Delta$. The explanation for these peculiarities is that the line segment between two points in stochastic diffusive steps does not tell us anything about intermediate locations of a particle. It could have been anywhere with a finite probability. In other words, sampling the path length or the estimator $\Phi$ does not allow to obtain the dwell time as it was possible by marker counting via Eq. 21. Therefore, a proper normalization is not provided. More details on the statistics of the estimator $\Phi$ compared to the jump length $|\Delta x|$ can be found in appendix $\mathrm{C}$. The only exception and possible application of the track length estimator $\Phi$ is in the pure drift which is deterministic and allows retroactive analysis of the straight line segment connecting two subsequent locations. But even this is not ensured if $V$ is not constant everywhere and certain errors occur if the time step is chosen too large. This will be discussed in more detail in the next section.

\section{THE 1D INHOMOGENEOUS CASE}

The discussion of section IV has shown that the details of the jump length in the drift-diffusive Monte-Carlo step do not give any advantage in the evaluation of the particles' trajectories. The clean representation of the dwell time is of central importance for the Monte-Carlo sampling, but can not be extracted from segments of the particle trajectories if diffusion is present. Rather, the trajectories represent only probabilities. An obvious problem related to the discussion of diffusion paths in section IV is: to what extent is the dwell time correctly taken into account by increasing the counter in the marker method by one, i. e. by using always the same weight even if diffusive detours are possible? This is of particular importance if a simulation particle starts close to a cell boundary and has a significant chance to get back to the starting cell after some detours in neighboring cells. This is illustrated in Fig. 2 where the dwell time $T_{i}\left(x_{0}\right)$ for a single time step $\Delta$ is shown as a function of the particle's starting position $x_{0}$. The dwell time $T_{i}\left(x_{0}\right)$ 
for each particular grid cell is introduced by

$$
T_{i}\left(x_{0}\right)=\int_{X_{i-1 / 2}}^{X_{i+1 / 2}} \int_{0}^{\Delta} G\left(x-x_{0}, t\right) d t d x
$$

with $X_{i-1 / 2}$ and $X_{i+1 / 2}$ denoting the location of the $i$ th cell boundaries. A dwell time $T_{i}\left(x_{0}\right) \approx \Delta$ means that the possible jumps of a particle started at a particular $x_{0}$ are most likely inside the $i$ th cell. A lower value means that the probability for detours into other cells is significant. It can be seen that particles starting in a particular cell in general have a finite dwell time in certain regions of the neighboring cells. This has to be taken into account when a particle step is to be weighted properly. Actu-

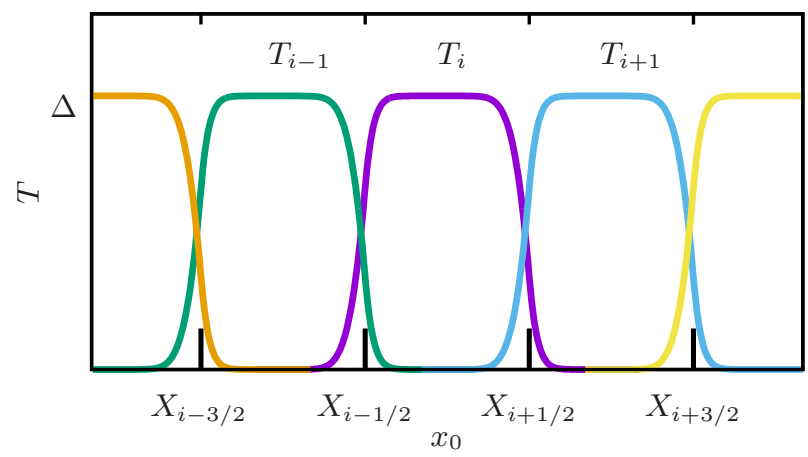

FIG. 2 Sketch of the dwell time $T_{k}$ for different cells as a function of the particle's starting position $x_{0}$. The time step is $\Delta$ and the dwell time can reach this value inside the cells if most possible steps are essentially inside a particular cell. But when the particle starts closer to the cell boundaries its dwell time in the neighboring cell is non-zero due to possible stochastic detours. The plots were obtained numerically by using the parameters $V=2 \mathrm{~m} / \mathrm{s}, D=1 \mathrm{~m}^{2} / \mathrm{s}, X_{i-3 / 2}=-1$, $X_{i-1 / 2}=2, X_{i+1 / 2}=5, X_{i+3 / 2}=8, \alpha=0$ and time step $\Delta=0.1$ $\mathrm{s}$.

ally, it is needed to consider the dwell time for each cell separately to gain information for the proper weighting of the Monte-Carlo sampling. On the other hand this means that the full Green's function integral Eq. 46 is evaluated and, indeed, this is what the Monte-Carlo algorithm is supposed to do. The situation becomes even worse when the inhomogeneous case with spatially varying velocity $V(x)$ and diffusion $D(x)$ is considered. Then the Green's function is not even known and the MonteCarlo stepping is justified only for regions with constant $V$ and $D$, as usual in discretized computational volumes. But, this leads directly to the requirement that the overlap in dwell times $T_{i}$ over cell boundaries as sketched in Fig. 2 should be as small as possible as it introduces numerical errors. To quantify the error one might estimate the width $b$ of the overlap in Fig. 2 where the dwell time $T_{i}$ drops from $\Delta$ to 0 . With the definition

$b\left|\frac{\partial T_{i}}{\partial x_{0}}\right|=\Delta$ and $\left|\partial T_{i} / \partial x_{0}\right|$ taken at a cell boundary, a measure for the error $E$ can be introduced by $E=b / \Delta_{x}$, where $\Delta_{x}=$ $X_{i}-X_{i-1}$ is the grid spacing of the computational grid. The requirement $b / \Delta_{x} \ll 1$ gives the condition

$E=\frac{\Delta}{\Delta_{x}}\left|\frac{\partial T_{i}}{\partial x_{0}}\right|_{i}^{-1} \ll 1$

taking $\left|\partial T_{i} / \partial x_{0}\right|_{i}$ as the minimum of $\left|\partial T_{i} / \partial x_{0}\right|$ at positions $X_{i-1 / 2}$ and $X_{i+1 / 2}$. The detailed expressions for those derivatives are given in appendix B.



FIG. 3 Sketch of the error estimates $E, E_{V=0}$ and $E_{D=0}$ defined in Eqs. 48, 49 and 50. The general error estimate $E$ combines the limiting cases $E_{V=0}$ and $E_{D=0}$ and is more restrictive for the choice of a time step $\Delta$. The plots were obtained numerically by using the parameters $V=2 \mathrm{~m} / \mathrm{s}, D=1 \mathrm{~m}^{2} / \mathrm{s}$, $\Delta_{x}=4 \mathrm{~m}$ and $\alpha=0$. Note that the curves of $E_{D=0}$ and $E_{V=0}$ always have two intersection points: one at $\Delta=0$ and the other at $\Delta=\pi D /\left(2|V|^{2}\right)$ and that for any finite values of $V$ and $D$ it follows that $E_{D=0}>E_{V=0}$ for $0<\Delta<\pi D /\left(2|V|^{2}\right)$.

It is important to note that for $\alpha=0$ the following limits of Eq. 48 can be found: For $V \neq 0$ and $D \rightarrow 0$

$E_{D=0}=|V| \frac{\Delta}{\Delta_{x}} \ll 1$

and for $V \rightarrow 0$ and $D>0$

$E_{V=0}=\sqrt{\frac{\pi D}{2 \Delta}} \frac{\Delta}{\Delta_{x}} \ll 1$

which are well known from stability analysis of finite difference schemes [17]. But here the conditions result from requirements on accuracy for the otherwise numerically stable Monte-Carlo procedure. The term stable in this context is used in the sense that no instabilities occur in the straightforward sampling along particle trajectories. Of course the application for linearized models of non-linear systems might suffer from the usual numerical difficulties with respect to unstable modes. In practice the error defined by the Eqs. 48-50 represents additional noise in the simulation results due to an improper weighting in the marker method when each particle increases the counter by one even if its cell specific weight might be much less than 1 at a location close to a cell boundary. 
This means that the instability which might appear as a problem in other numerical methods due to large time steps becomes a problem with a lower limit of noise in the Monte-Carlo procedure. This minimum level of noise increases with time step. The Fig. 3 illustrates the condition of Eq. 48 in comparison with the limiting cases of Eqs. 49 and 50. To extend the discussion of time stepping the estimates for numerical errors introduced by the dwell time $T_{i}$ in Eq. 46 for each cell are applied for the case of varying drift and diffusion coefficients $V(x)$ and $D(x)$. To derive a general expression of the Green's function even for the case of piecewise constant coefficients is a complicated task (see e. g. [18-20]). Therefore, we give a more crude estimate of this problem by employing again the approximative expression of Eq. 46. First we note that insertion of the limiting cases expressed by Eqs. 49 and 50 into Eq. 38 gives immediately $L(\Delta) \ll \Delta_{x}$ for $D \rightarrow 0$ and $L(\Delta) \ll 2 \Delta_{x} / \pi$ for $V \rightarrow 0$. Therefore, the time step constraint derived from the requirement of reduced Monte-Carlo noise can be reformulated as a constraint for the average jump length $L(\Delta)$ defined in Eq. 38. The result $L(\Delta) \ll \Delta_{x}$ is true also for the general numerical error of Eq. 48. Stressing even further the argument of small overlap width $b$ as defined by Eq. 47 one might consider an error due to different values of $V$ and $D$ in neighboring cells by the scaled difference

$E^{*}=2 \frac{b_{\mathrm{I}}-b_{\mathrm{II}}}{b_{\mathrm{I}}+b_{\mathrm{II}}}$

where $b_{\mathrm{I}}$ and $b_{\mathrm{II}}$ are the width of the overlapping region for a single interface where $V=V_{\mathrm{I}}$ and $D=D_{\text {I }}$ on the one side of the interface and $V=V_{\text {II }}$ and $D=D_{\text {II }}$ on the other. Using the formulas of Eqs. B5 and B6 given in the appendix B for a single interface (formally: $\Delta_{x} \rightarrow 0$ ) one obtains

$b_{\mathrm{I}}=\frac{\left|V_{\mathrm{I}}\right| \Delta}{\operatorname{erf}\left(\frac{\left|V_{\mathrm{I}}\right| \Delta}{\sqrt{2 D_{\mathrm{I}} \Delta}}\right)} \quad, \quad b_{\mathrm{II}}=\frac{\left|V_{\mathrm{II}}\right| \Delta}{\operatorname{erf}\left(\frac{\left|V_{\mathrm{II}}\right| \Delta}{\sqrt{2 D_{\mathrm{II}} \Delta}}\right)}$

To simplify matters even more the error $E^{*}$ is approximated by

$E^{*} \approx \Delta_{x} \frac{\partial b / \partial x}{b} \quad, \quad b=\frac{|V| \Delta}{\operatorname{erf}\left(\frac{|V| \Delta}{\sqrt{2 D \Delta}}\right)}$,

where $\Delta_{x}$ denotes again the (finite) cell width, to obtain

$E^{*}=\frac{\Delta_{x}}{L_{V}}-\left(\frac{\Delta_{x}}{L_{V}}-\frac{\Delta_{x}}{2 L_{D}}\right) \sqrt{\frac{2 b^{2}}{\pi D \Delta}} \exp \left(-\frac{V^{2} \Delta}{2 D}\right)$

with gradient lengths of $V$ and $D$ defined by

$L_{V}=\frac{V}{\partial V / \partial x} \quad, \quad L_{D}=\frac{D}{\partial D / \partial x}$

For $\Delta \rightarrow 0$ one finds $E^{*}=\Delta_{x} /\left(2 L_{D}\right)$ and for $\Delta \rightarrow \infty$ the limit is $E^{*}=\Delta_{x} / L_{V}$. For arbitrary time steps $\Delta$ the values of $E^{*}$ are between these two limits. Not surprisingly, the requirement $E^{*} \ll 1$ leads to the the constraint $\Delta_{x} \ll L_{V}, L_{D}$, meaning that the computational grid must be capable of resolving the spatial variations in the coefficients $V$ and $D$ properly. Altogether, the requirements on accuracy and noise reduction in Fokker-Planck Monte-Carlo simulations, i. e. $E \ll 1$ and $E^{*} \ll 1$, give constraints for the time step $\Delta$ and the grid resolution $\Delta_{x}$ in the marker method which are identical to explicit finite difference methods.

\section{CONCLUSION}

The problem of Monte-Carlo time stepping algorithms for Fokker-Planck equations has been reconsidered. The basic objective in finding numerical solutions of stationary problems is the numerical estimation of the dwell time. This can be obtained by the standard procedure of launching an ensemble of simulation particles and tracking their entire paths by counting markers in a discretized grid. The ratio of the cumulative number of particles and the number of launched particles per time step provides the dwell time and a proper normalization to connect the simulation results with the physical system under consideration. The path length sampling has been considered as a possible alternative for the time consuming counting of markers. This is obviously a simple and efficient method for free flight problems, like for neutral particles or neutrons in many applications, where the motion is dominated by drift and therefore is deterministic. However, it is shown that the stochastic nature of the diffusive piece in the transport excludes the use of simple track length sampling because the path length of diffusion trajectories is not a well defined quantity and not appropriate to represent the dwell time. As long as no other track length based estimator is found - and in this work no proposal is given - the stepping algorithm is restricted to the usual marker method. An additional analysis of the inhomogeneous $1 \mathrm{D}$ problem allowed to derive further restrictions on the construction of Monte-Carlo trajectories which shows that the well known Courant-FriedrichsLewy condition for explicit finite difference schemes has to be taken into account also for the marker based MonteCarlo schemes.

\section{Acknowledgments}

This work has been carried out within the framework of the EUROfusion Consortium and has received funding from the Euratom research and training programme 2014-2018 under grant agreement No 633053. The views and opinions expressed herein do not necessarily reflect those of the European Commission. 


\section{APPENDIX A: Dwell time and other averages in the} continuous limit

The dwell $\tau_{m}$ has been defined in section II by the discrete expression Eq. 15

$$
\tau_{m}=\frac{\sum_{i=1}^{m} \int_{\Omega} t_{i} f^{-}\left(\mathbf{x}, t_{i}\right) d \mathbf{x}}{\sum_{i=1}^{m} \int_{\Omega} f^{-}\left(\mathbf{x}, t_{i}\right) d \mathbf{x}}
$$

Assuming that all requirements of measure theory and Lebesgue integration are met one can introduce a probability measure $\mu$

$$
\begin{aligned}
\mu\left(t_{m}\right) & =\sum_{i=1}^{m} \int_{\Omega} f^{-}\left(\mathbf{x}, t_{i}\right) d \mathbf{x} \\
& =1-\int_{\Omega} f^{+}\left(\mathbf{x}, t_{m}\right) d \mathbf{x} \equiv \int_{0}^{t_{m}} \mu^{\prime}(t) d t
\end{aligned}
$$

with density $\mu^{\prime}$

$\mu^{\prime}(t)=-\int_{\Omega} \frac{\partial f^{+}(\mathbf{x}, t)}{\partial t} d \mathbf{x}$

Then the dwell time $\tau_{m}$ in the continuous limit is written as

$\tau_{m}=\int_{0}^{t_{m}} t \frac{\mu^{\prime}(t)}{\mu\left(t_{m}\right)} d t$

This can be evaluated via

$$
\begin{aligned}
& \int_{0}^{t_{m}} t \mu^{\prime}(t) d t=-\int_{\Omega} \int_{0}^{t_{m}} t \frac{\partial f^{+}(\mathbf{x}, t)}{\partial t} d t d \mathbf{x} \\
& \quad=-t_{m} \int_{\Omega} f^{+}\left(\mathbf{x}, t_{m}\right) d \mathbf{x}+\iint_{\Omega} \int_{0}^{t_{m}} f^{+}(\mathbf{x}, t) d t d \mathbf{x}
\end{aligned}
$$

to obtain

$$
\begin{aligned}
\tau_{m}- & \tau_{m} \int_{\Omega} f^{+}\left(\mathbf{x}, t_{m}\right) d \mathbf{x} \\
& =\int_{\Omega} \int_{0}^{t_{m}} f^{+}(\mathbf{x}, t) d t d \mathbf{x}-t_{m} \int_{\Omega} f^{+}\left(\mathbf{x}, t_{m}\right) d \mathbf{x}
\end{aligned}
$$

The stationarity of the problem considered here requires that $f^{+}\left(\mathbf{x}, t_{m}\right)=0$ for $t_{m}>t_{M}$, with a finite time $t_{M}$ giving the upper limit for a Monte-Carlo chain. Then one finds for $\tau=\lim _{t_{m} \rightarrow \infty} \tau_{m}$

$\tau=\int_{\Omega} \int_{0}^{\infty} f^{+}(\mathbf{x}, t) d t d \mathbf{x} \equiv \int_{\Omega} F^{+}(\mathbf{x}) d \mathbf{x}$

In a similar way one can derive the chain average $\omega_{m}$ of a quantity $w=w(t)$

$\omega_{m}=\frac{\sum_{i=1}^{m} \int_{\Omega} w\left(t_{i}\right) f^{-}\left(\mathbf{x}, t_{i}\right) d \mathbf{x}}{\sum_{i=1}^{m} \int_{\Omega} f^{-}\left(\mathbf{x}, t_{i}\right) d \mathbf{x}}$

with the continuous limit

$\omega=\int_{\Omega} \int_{0}^{\infty} \frac{\partial w}{\partial t} f^{+}(\mathbf{x}, t) d t d \mathbf{x}$

\section{APPENDIX B: Evaluation of derivatives in the time stepping} error

The definition of Eq. 46 for the dwell time $T_{i}$ in the $i$ th cell

$T_{i}=\int_{X_{i-1 / 2}}^{X_{i+1 / 2}} \int_{0}^{\Delta} G\left(x-x_{0}, t\right) d t d x$

gives

$\frac{\partial T_{i}}{\partial x_{0}}=\int_{0}^{\Delta} G\left(X_{i-1 / 2}-x_{0}, t\right) d t-\int_{0}^{\Delta} G\left(X_{i+1 / 2}-x_{0}, t\right) d t$

This can be evaluated using the relation

$$
\begin{aligned}
& \int_{0}^{\Delta} G(x, t) d t \\
& \quad=\frac{1}{2 v^{\prime}} \exp \left(\frac{v x}{D}-\frac{v^{\prime}|x|}{D}\right)\left[1+\operatorname{erf}\left(\frac{v^{\prime} \Delta-|x|}{\sqrt{2 D \Delta}}\right)\right] \\
& \quad-\frac{1}{2 v^{\prime}} \exp \left(\frac{v x}{D}+\frac{v^{\prime}|x|}{D}\right)\left[1-\operatorname{erf}\left(\frac{v^{\prime} \Delta+|x|}{\sqrt{2 D \Delta}}\right)\right]
\end{aligned}
$$

where

$v^{\prime}=\sqrt{2 \alpha D+v^{2}}$ 
Defining $\Delta_{x}=X_{i+1 / 2}-X_{i-1 / 2}$ one finds for $x_{0}=X_{i-1 / 2}$

$$
\begin{aligned}
& \left.\frac{\partial T_{i}}{\partial x_{0}}\right|_{X_{i-1 / 2}}=\frac{1}{v^{\prime}} \operatorname{erf}\left(\frac{v^{\prime} \Delta}{\sqrt{2 D \Delta}}\right) \\
& -\frac{1}{2 v^{\prime}} \exp \left(\frac{v \Delta_{x}}{D}-\frac{v^{\prime} \Delta_{x}}{D}\right)\left[1+\operatorname{erf}\left(\frac{v^{\prime} \Delta-\Delta_{x}}{\sqrt{2 D \Delta}}\right)\right] \\
& +\frac{1}{2 v^{\prime}} \exp \left(\frac{v \Delta_{x}}{D}+\frac{v^{\prime} \Delta_{x}}{D}\right)\left[1-\operatorname{erf}\left(\frac{v^{\prime} \Delta+\Delta_{x}}{\sqrt{2 D \Delta}}\right)\right]
\end{aligned}
$$

and at $x_{0}=X_{i+1 / 2}$ the derivative is

$$
\begin{aligned}
& \left.\frac{\partial T_{i}}{\partial x_{0}}\right|_{X_{i+1 / 2}}=-\frac{1}{v^{\prime}} \operatorname{erf}\left(\frac{v^{\prime} \Delta}{\sqrt{2 D \Delta}}\right) \\
& +\frac{1}{2 v^{\prime}} \exp \left(-\frac{v \Delta_{x}}{D}-\frac{v^{\prime} \Delta_{x}}{D}\right)\left[1+\operatorname{erf}\left(\frac{v^{\prime} \Delta-\Delta_{x}}{\sqrt{2 D \Delta}}\right)\right] \\
& \quad-\frac{1}{2 v^{\prime}} \exp \left(-\frac{v \Delta_{x}}{D}+\frac{v^{\prime} \Delta_{x}}{D}\right)\left[1-\operatorname{erf}\left(\frac{v^{\prime} \Delta+\Delta_{x}}{\sqrt{2 D \Delta}}\right)\right]
\end{aligned}
$$

\section{APPENDIX C: Statistics of the estimator $\Phi$}

To be more precise and pay sufficient attention to the random character also present in the evolution of the estimator $\Phi_{i}$ we will consider more in detail the properties of the scaled path length. According to Eq. 42 the MonteCarlo step for the estimator $\Phi$ for time step $\Delta$ is

$\Phi=\frac{|\Delta x|}{L(\Delta)} \Delta=\left|\frac{V}{L(\Delta)} \Delta^{2}+\gamma \frac{\sqrt{D \Delta}}{L(\Delta)} \Delta\right|$

Due to the normal distribution of $\Delta x$ with mean $\langle\Delta x\rangle=$ $V \Delta$ and variance $\left\langle(\Delta x)^{2}\right\rangle-\langle\Delta x\rangle^{2}=D \Delta$ the absolute value $|\Delta x|$ obeys a folded normal distribution with mean and variance

$$
\langle|\Delta x|\rangle=L(\Delta)
$$

$$
\left\langle|\Delta x|^{2}\right\rangle-\langle|\Delta x|\rangle^{2}=(V \Delta)^{2}+D \Delta-L(\Delta)^{2}
$$

Consequently the distribution of $\Phi$ is also folded normal Its mean $\langle\Phi\rangle$ is independent of $V$ and $D$ and as expected

$$
\langle\Phi\rangle=\Delta
$$

and its variance is

$$
\left\langle\Phi^{2}\right\rangle-\langle\Phi\rangle^{2}=\frac{V^{2} \Delta^{4}}{L(\Delta)^{2}}+\frac{D \Delta^{3}}{L(\Delta)^{2}}-\Delta^{2}
$$

It is interesting to compare the limiting case $V=0$ and finite $D$ and vice versa. For $V \neq 0$ and $D=0$ :

$\langle|\Delta x|\rangle=|V| \Delta$

$\left\langle|\Delta x|^{2}\right\rangle-\langle|\Delta x|\rangle^{2}=0$

$\left\langle\Phi^{2}\right\rangle-\langle\Phi\rangle^{2}=0$

and for $V=0$ and $D \neq 0$ :

$\langle|\Delta x|\rangle=\sqrt{\frac{2 D \Delta}{\pi}}$

$\left\langle|\Delta x|^{2}\right\rangle-\langle|\Delta x|\rangle^{2}=\left(\frac{\pi}{2}-1\right) \frac{2 D \Delta}{\pi}$

$\left\langle\Phi^{2}\right\rangle-\langle\Phi\rangle^{2}=\left(\frac{\pi}{2}-1\right) \Delta^{2}$

One finds that for pure diffusive motion the variance of $\Phi$ becomes independent of $D$ and also the dynamics becomes super-diffusive. This is the most important difference compared to the statistics of $|\Delta|$. There the variance for pure diffusion is proportional to the time step $\Delta$ as it is also for $\Delta x$. But $\Phi$ goes with $\Delta^{2}$ in that case.

\section{References}

[1] Binder, K., "Applications of the Monte Carlo Method in Statistical Physics", Topics in Current Physics Vol. 36, Springer-Verlag Berlin Heidelberg, 1987

[2] Kloeden, P. E., Platen, E. and Schurz, H., "Numerical Solution of SDE through Computer Experiments", Springer-Verlag Berlin Heidelberg, 1994

[3] Graham, C. and Talay, D., "Stochastic Simulation and Monte Carlo Methods", Stochastic Modelling and Applied Probability Vol. 68, Springer-Verlag Berlin Heidelberg, 2013

[4] Kalos, M. H. and Whitlock, P. A., "Monte Carlo Methods", Wiley-VCH, 2008

[5] Kroese, D. P. et al., "Why the Monte-Carlo method is so important today", WIREs Comput Stat 2014, 6:386-392

[6] Hakola, A. et al., Plasma Phys. Control. Fusion 55, 124029 (2013)

[7] Stangeby, P. C. and Elder, J. D., J. Nucl. Mater. 196-198, p. $258(1992)$

[8] Hirvijoki, E. et al., Journal of Plasma Physics 81, 435810301 (2015)

[9] Shimizu, K. et al., Nucl. Fusion 49065028 (2009)

[10] Reiter, D., J. Nucl. Mater. 196-198, p. 80 (1992)

[11] Wieggers, R. et al., J. Nucl. Mater. 438, p.643 (2013)

[12] Heifetz, D. et al., J. Comp. Phys. 46309 (1982)

[13] Feng Y. et al., J. Nucl. Mater. 241-243, 930 (1997)

[14] Spanier, J. and Gelbard, E. M., "Monte Carlo Principles and Neutron Transport Problems", Dover Publications, 2008

[15] Risken, H., "The Fokker-Planck Equation", Springer Series in Synergetics Vol. 18, Springer-Verlag Berlin Heidelberg, 1996 
[16] Gardiner, C., "Stochastic Methods", Series in Synergetics Vol. 13, Springer-Verlag Berlin Heidelberg, 2009

[17] Kajishima, T. and Taira, K., "Computational Fluid Dynamics", Springer International Publishing 2017

[18] Ramirez, J. M., Thoman, E. A. and Waymire, E. C.,
Statistical Science, Vol. 28, No. 4, 487-509 (2013)

[19] Lejay, A., Probability Surveys Vol. 3, 413-466 (2006)

[20] Lejay, A., Elect. Comm. in Probab. 16, 764-774 (2011) 